\title{
Collaboration Management System between the Device based on Machine Socialization
}

\author{
Han Kyung Kim, In Shik Kang, Wung Jun Kim, Hoe Kyung Jung \\ Department of Computer Engineering, PaiChai University, Doma2-Dong, SeoGu, DaeJeon, Korea
}

\begin{tabular}{l}
\hline \hline Article Info \\
\hline Article history: \\
Received Aug 12, 2016 \\
Revised Oct 25, 2016 \\
Accepted Nov 8, 2016 \\
\hline
\end{tabular}

Keyword:

IoT

Machine socialization

Micro controller

Schema

Sensor

\begin{abstract}
The basis of IoT is in the interconnection and communication between different devices to achieve common goals through internet. These devices are interconnected through a network which enables communication within these devices without any direct human intervention. But with such great potential, this technology reached a road-block due to incompatibility within various manufacturers of the same type of device and proprietary standards. I started this project with this problem in mind and I have created a brand and platform independent machine socialization device manager system. In this paper, to overcome the above mentioned problem, I have utilized micro controllers to connect to various existing device to solve the problem and propose a device to device communication with collaboration management. This technology is not restricted to usage in only the new network module enabled smart devices but also this can be used to operate the existing old (not smart) home appliances. Machine socialization was made possible with the use of XML, (an internet standard schema language) which we have used to gather device, task and relationship information of all the devices to show schema information.
\end{abstract}

Copyright $\odot 2016$ Institute of Advanced Engineering and Science. All rights reserved.

\section{Corresponding Author:}

Hoe Kyung Jung,

Department of Computer Engineering,

Paichai University,

155-40 University Road, Seogu, DaeJeon, Korea.

Email: hkjung@pcu.ac.kr

\section{INTRODUCTION}

The IoT (Internet of Things) was developed as a medium of communication between a variety of physical objects around us [1-2]. This has made connectivity between various devices possible in the same environment, and devices share information like in smart home, smart car and health care. IoT is implemented in the introductory stage and in development stage smart grid services/management, security, communications infrastructure, the Sensor and Cloud connection, such as a variety of studies are implemented. Companies are now using various smart appliances according to the development of IoT. They release devices and take smart appliances in its developing platform for these devices [3]. Research groups around the world are working on standardizing the IoT and companies like Qualcomm's representative AllJoyn are coming together for the same [4]. Accordingly, there are numerous products and platform to be released, and it is possible for the same platform device groups to communicate but there is a problem to communicate between different platforms.

In this paper, we propose a machine socialization device management system which enables communication between different platforms devices. For this purpose, a small embedded system Arduino combines General electronics products and circumstantial power control, and OpenWRT AP server using the schema and build the Device Manager and cross-compatible sensors or user coming from the device 
depending on the event. The purpose of this machine socialization device system for managing the collaboration between the device providing a customized service through the relationship between devices.

\section{THE APPLIANCE IS DESIGNED FOR CROSS-COLLABORATION MANAGEMENT SYSTEM}

Machine socialization device system is based on devices collaborative management system proposed is composed of event processing and storing the information in a schema, database, equipment and general manager of devices collaborative task manager, under the command driven products to start work.

In the device management system, among the many methods used for building a wired/wireless router, the server assigns the IP devices built after coming from the sensor value, the return value is stored in the database, schema, and PHP is configured as Device Manager are inherent in the format. Papers suggested system configuration is shown in Figure 1.

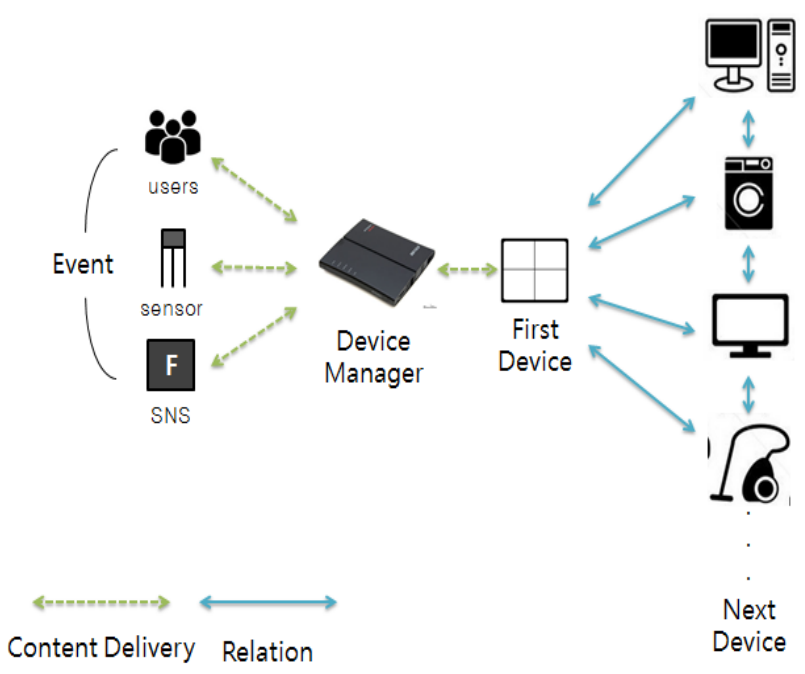

Figure 1. Block Diagram of Device Manager

Machine socialization schema devices uses information such as an identification number, function descriptions, hours of operation of the device in the Device_info information and identification numbers, the working group the working group description, work priorities, and decides what to do with the relationship between the different gadgets function the appliance's and Task_info, define a Relation is divided into.

This scheme based on the defined event occurs and the data is passed to the server in the router's home, located on the server appliance administrator should run the scenarios to find a job for your event in Task_info.

Each scenario is a priority and when it overlaps several operations to give priority to. Running the scenario Device_info based on the information appliance the operation is carried out with the information in accordance with the order.

Running the scenario Device_info based on the information appliance in operation is carried out with the information in accordance with the order. These events are the user's current situation and the situation within the home, the devices on the server and sent to the administrator.

Device Manager is based on the context data sent to the device control operations by providing services to users. Figure 2 shows the architecture of the machine indicates the social applications.

In these papers I offer in collaboration between the appliance management system to implement a prototype application of wired and wireless network access devices. But the current consumer electronics market, the price of the products are expensive and each possible networking its way in tandem with the platform to fit because the fixed price is expensive and each of its platform for the indexation of the products are not suitable.

Therefore, these systems typically use a micro-controller devices to connect Arduino Uno Board Wi-Fi shield attached to the server and data communication to, Arduino and equipment between relay (SSR: Solid State Relay) is used to allow you to control the power. Figure 3 is an Arduino board to take advantage of the product indicates the flow control. 


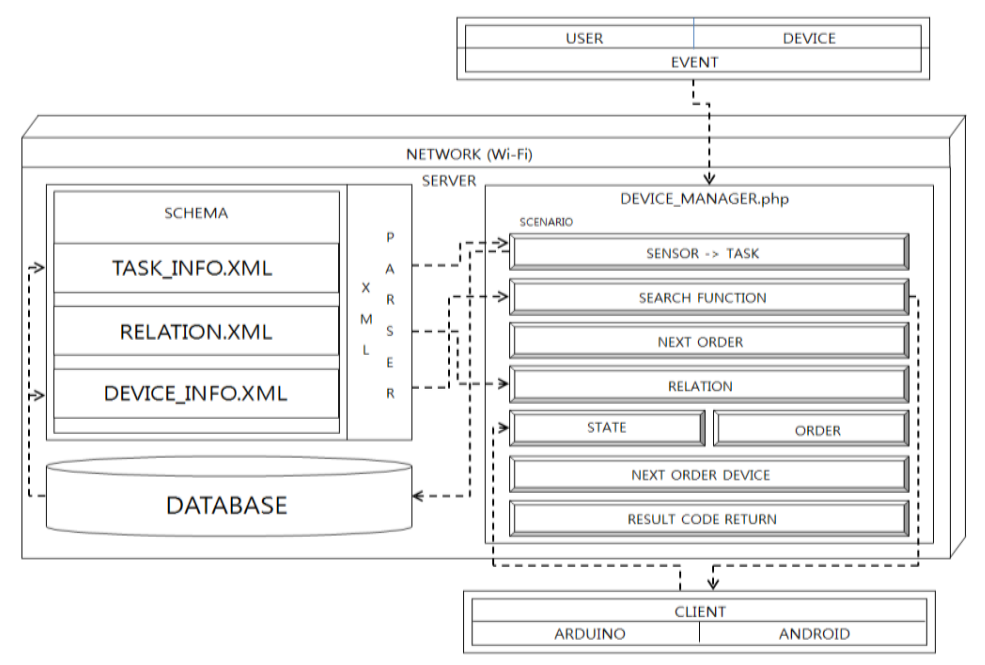

Figure 2. Architecture of Machine Socialization System

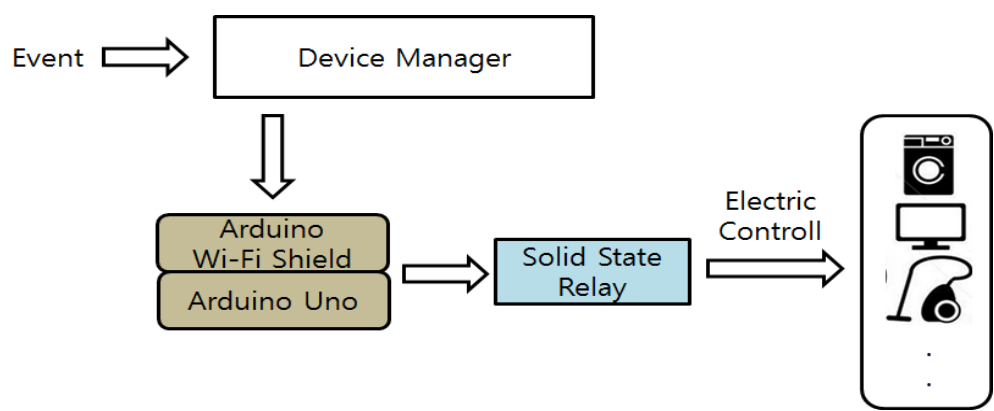

Figure 3. Flow Chart of Device Electric Control

\section{EQUIPMENT MANAGEMENT SYSTEM IMPLEMENTATION}

Device management system among the many methods for building a router, leave the server in PHP on the server by assigning IP, consisting of a type designed for appliance administrators. Before you run the device Device_info devices to check the device status information and to determine the priority for execution. When the execution of the first unit is complete, the device manager then has to find the relationship between the unit and other components are complete, navigate to the device operation to be executed in the following, and then re-check the machine's status and priority in Device_info.

In this environment, a cross-platform device that works using the Arduino limitations in the operating system. To win social applications, and consumer electronics products AP schema Arduino boards, Arduino UNO Wi-Fi shield, sensor, relay is connected to the operation. To manage the server and the equipment through machines socialization device management schema to the AP schema Arduino boards, Arduino UNO Wi-Fi shield, sensor, relay is connected to the operation. User with the device or within the device family most closely related with the ambient light sensor, an acceleration sensor, a gyro sensor, etc. and provides the event.

These events are the user's current situation and the situation within the home, the devices on the server and sent to the administrator. Device Manager is based on the received data sent through the process conducted by a context-sensitive device control provides to the user.

Table 1 is a development environment in the collaboration between the device management system, Figure 4 and Figure 5 is a flow chart showing the operation of the prototype and scenario. 
Table 1. Example of Mapping Rule

\begin{tabular}{ll}
\hline Tools & Edit Plus, Arduino Sketch, APMsetup 7 \\
\hline PC & Window \& Enterprise \\
& Intel i5 - 3450, 8GB RAM \\
& Fan, Vacuum Cleaner, Mini Motor, \\
Device & Arduino Uno,Arduino Wi-Fi Shield, \\
& Bread Board, Temperature Sensor, SSR \\
\hline
\end{tabular}

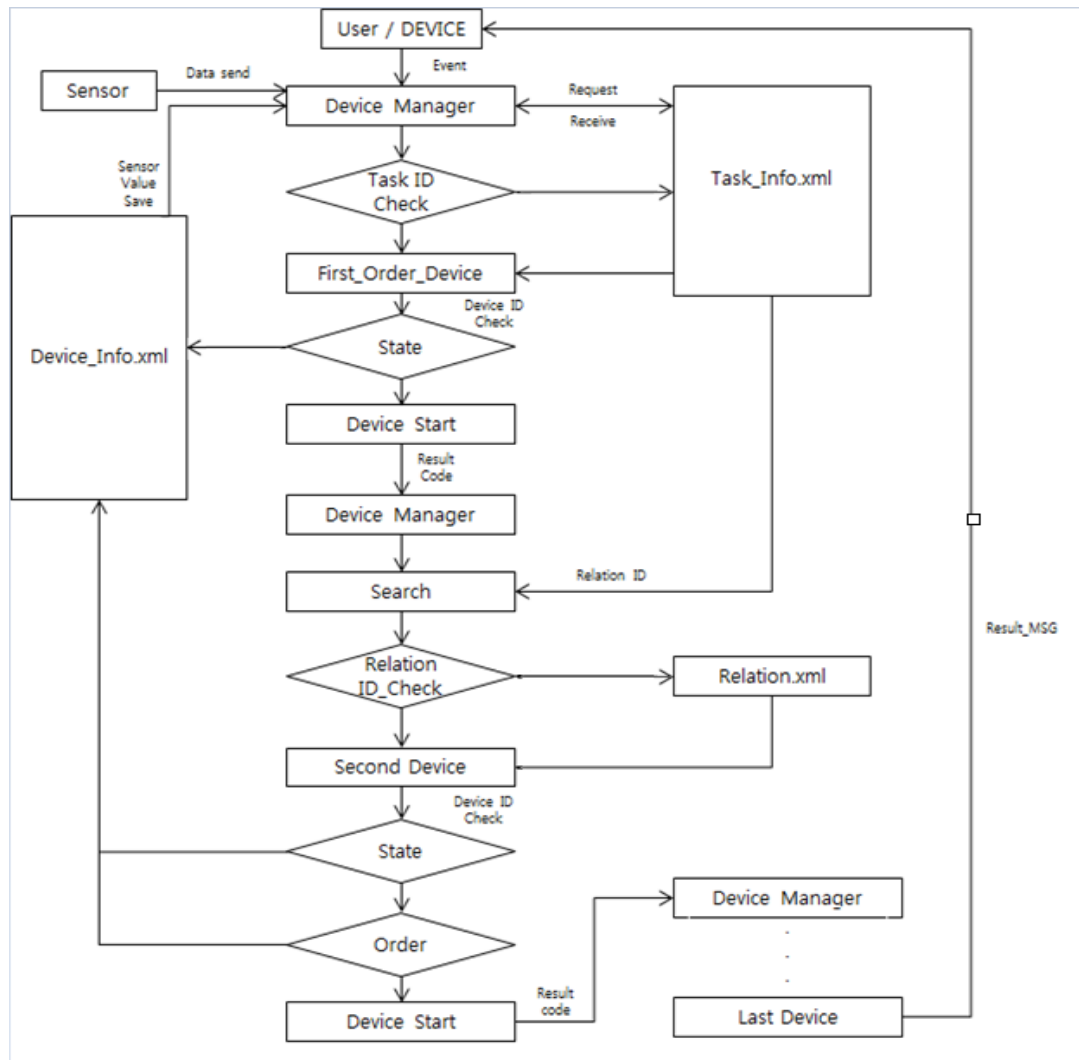

Figure 4. Prototype Flow Chart

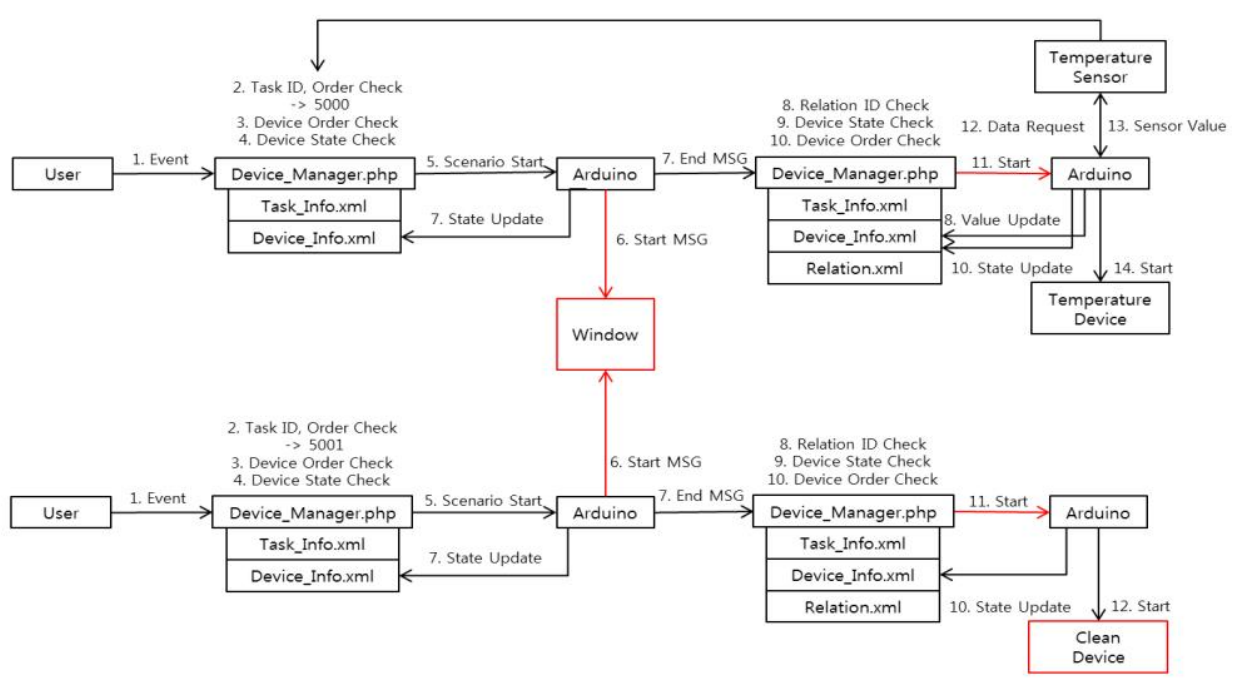

Figure 5. Flow Prototype Scenario 
Temperature control operation for controlling the temperature of the house at a temperature preferred by the user operation and a cleaning operation for cleaning automatically divided into the set time was designed to house the two operations overlap in the windows device. The temperature values measured by the sensors are stored in a DB by the device administrator and stored in Device_Info back up the values in the DataBase.

Device Manager generates an event for the temperature control and work with the stored temperature value. The cleaning process between the two priority tasks to be executed when the appropriate time is set higher than the cleaning process. When the execution is completed, each device will return to the Result_Code report this progress to the next task. Figure 5 shows a prototype workflow. The prototype apparatus in applying the system window, fans, vacuum cleaners, and a temperature sensor. The temperature regulating functions and operations with the closure of the fans and window cleaning operations with the opening function and window cleaner. Temperature control work has been replaced by a fan attached to one temperature sensor suitable to use air conditioning. It is suitable for machines to show the collaboration between socialization devices that states because one of the devices to perform different functions into two jobs. Arduino connect the relay between board and machine. Arduino begins to deliver the command to the electric signal of $5 \mathrm{~V}$ relay to accept the electrical signals of the device $220 \mathrm{~V}$ in the machine receives the electrical signal of $5 \mathrm{~V}$. If the input signal and load side of the relay are electrically isolated and optical signal is simplified because only delivered part considers the counter-electromotive force or electrical isolation of the load circuit by side with fewer coils.

\section{CONCLUSION AND FUTRUE RESEARCH}

In this paper, in order to start the operation the events to device manager in the server in the present system to start a temperature control operation was specified, the value coming from a temperature sensor, using the method if the predetermined period of time to send a signal to begin the cleaning process the people of the automated system was implemented using data without direct command of the instrument. The system is to be recognized by the external control, as well as home appliances such as the sensor and the time the device itself is a status management unit by providing efficient collaboration between the ability to operate the work. Relationship between devices are set and the information based on the user preference are set without human intervention.

If the information about the schema specification is correct in the server, regardless of platform type of product is expected to achieve common goals. Irrespective of the platform the product within the server is equipped with the appropriate information in the schema-compliant if the system brings good things because it can be done in the Internet environment is expected to continue.

\section{ACKNOWLEDGEMENTS}

This work was supported by the research grant of Pai Chai University in 2016.

\section{REFERENCES}

[1] M Bergman, "Consumer Electronics Magazine," Product and Technology Trends from the 2015 International CES, IEEE, 2015.

[2] J. Kang, et al., "Market and Technical Trends of internet of things," The Korea Contents Society, vol/issue: 13(1), pp. 14-17, 2015.

[3] J. H. Kang, et al., "A Patent Trend Analysis for Technological Convergence of IoT and Wearables," Korean Institute of Intelligent Systems, vol/issue: 25(3), pp. 306-311, 2015.

[4] P. Pande and A. R. Padwalkar, "Internet of Things-A Future of Internet: A Survey," International Journal, 2014. 


\section{BIOGRAPHIES OF AUTHORS}
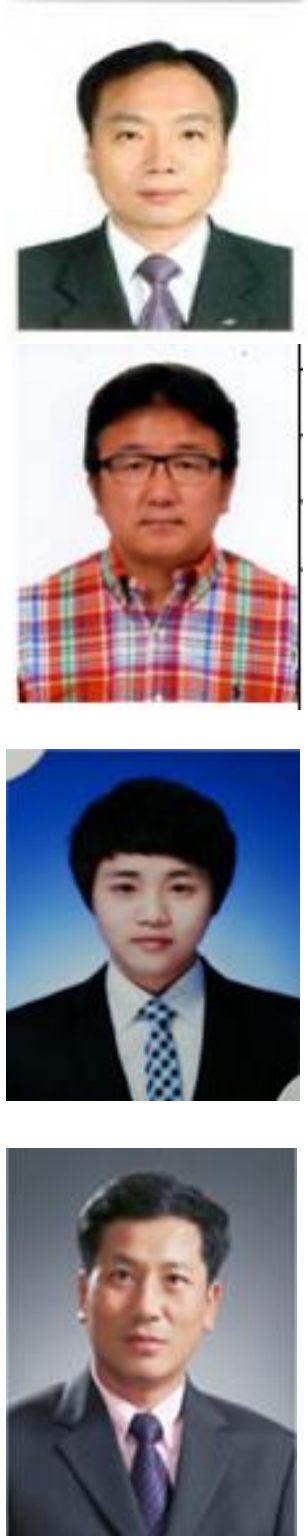

Han Kyung Kim. He received the M.S. degree in 2007 from theDepartment of Computer Communications Engineering of ChungbukNational University, Korea. Since 1989, he has worked at K-water (Korea Water Resources Company), where he now works as aDirector General in the Department of Water Information \&Technology Innovation. His current research interests include IoT (Internet of Things) of water resources field, ICT infrastructureand databases.

In Shik Kang. He received the B.S. degrees from the Department of Drama and Cinema of Cheongju University, Korea, in 1992, Since 2013, he worked for the Department of Music \& Sound Technology at Korea University of Media Arts as a professor. He is currently a Master course in Department of Computer Engineering of Paichai University. His current research interests include video signal, audio signal, IS.

Wung Jun Kim. He received the Bachelor of Engineering and Master's degrees from the Department of Computer Engineering of Paichai University, Korea, in 2014 and 2016, respectively. His current research interests app application(Android), IoT(Internet of Things) and M2M.

Hoe Kyung Jung. He received the M.S. degree in 1987 and Ph. D. degree in 1993 from the Department of Computer Engineering of Kwangwoon University, Korea. From 1994 to 1995 , he worked for ETRI as a researcher. Since 1994, he has worked in the Department of Computer Engineering at Paichai University, where he now works as a professor. His current research interests include multimedia document architecture modeling, information processing, information retrieval, and databases. 